\title{
L'ASSASSINAT DU SAVANT ABŪ MARWĀN AL-ṬUBNİ: DRAME FAMILIAL ET JUDICIAIRE ${ }^{1}$
}

\author{
CHRISTIAN MÜLLER \\ IRHT-CNRS, París
}

Le matin du 12 rabi $^{-}$II 457 / 23 mars 1065, des cris de surprise et de rage alertent le voisinage dans un quartier résidentiel de la ville de Cordoue. L'horrible nouvelle se répand: le savant Abū Marwān 'Abd al-Malik al-Ṭubnī a été trouvé par les femmes de sa maison poignardé dans son lit. Né en 396/1006, il avait dépassé les 58 ans. Par la suite, son fils ainé nommé Ziyādat Allāh annonça le meurtre de son père au prince de la ville Ibn Ğahwar en portant le deuil blanc habituel.

Abū Marwān al-Ṭubnī était une personne publique connue dans la ville: grand expert en philologie ('ilm al-luga) et langue arabe des bédouins (al-'arabiyya), il avait acquis pendant deux voyages dans l'Orient musulman la réputation de transmetteur de traditions prophétiques et de récits transmis par des savants (hadīt wa-riwāyāt). Après son retour des pays lointains de l'Est du monde musulman, il retrouva un nombre impressionnant de disciples autour de lui ${ }^{2}$. Son mode de vie, en revanche, restait très modeste, soulignant son indépendance matérielle face au pouvoir politique. Ainsi, il refusa un généreux salaire mensuel que lui proposait le souverain Ibn Ğahwar ${ }^{3}$ et n'accepta que des vêtements simples et de quoi ne pas mourir de faim, ce qui

1 Cet article est une version élargie et remaniée d'une analyse du cas dans mon Gerichtspraxis im Stadtstaat Cordoba, Leyde 1999, 320-31. Je remercie mes amis et collègues Jacqueline Sublet et Jean-Pierre Molénat qui ont revu mon texte sur le plan stylistique.

2 Humaydī, Ğadwat al-muqtabis, Le Caire, $1966\left(=(\mathrm{H}(\mathrm{C})), \mathrm{n}^{\circ}\right.$ 629, 285, cité aussi par Ibn Baškuwāl, Kitāb al-Șila, Le Caire, 1955 (= (IB(C)), n. ${ }^{\circ} 772,344$. Son influence dans la longue durée était peut être moins importante: étant donné sa position dans le réseau de savants et de la transmission, Dominique Urvoy lui accorde une importance moyenne: Urvoy, D., Le monde des ulémas andalous du $V^{e} / X I^{e}$ au VII $/ X I I I^{e}$ siècle. Étude sociologique, Genève, 1978, 96 sq., ainsi idem, El mundo de los ulemas andaluces del siglo V/XI al VII/XIII. Estudio sociologico, Madrid, 1983, 112 sq.

3 Abū 'l-Walīd b. Ğahwar, prince de Cordoue entre $435 / 1043$ et $461 / 1069$.

Al-Qanțara XXVI, 2 (2005) 425-448 
étonnait tout le monde ${ }^{4}$. Connu pour son avarice qui était devenue proverbiale dans la ville, on peut se demander si son refus ostensible d'accepter l'argent du souverain n'avait pas pour but d'accroître sa réputation de savant. En tout cas, il n'avait pas besoin d'un apport financier pour nourrir sa famille. Il vivait dans une grande maison $(d \bar{a} r)$ dans le quartier de la Mosquée al-Amir, situé à l'intérieur de la muraille dans la partie Est (al-ǧānib al-šarqĩ, Ajarquía) de la ville de Cordoue 5 .

Le savant assassiné appartenait à un clan illustre dont les membres étaient venus à plusieurs reprises au siècle précédent de la ville de Tubna, située dans la région du Zāb (dans le nord-est de l'Algérie actuelle), à la cour des Ommeyades à Cordoue. Plusieurs membres du clan occupaient de hautes charges officielles; parmi eux des poètes et des savants réputés au Vème/XIème siècle sous les Banū Ğahwar. II existait donc des relations personnelles entre la famille de la victime et le prince de la ville, ce qui pourrait expliquer certains aspects de l'affaire ${ }^{6}$.

Dès l'annonce du meurtre, le fils fut assailli de questions par des gens consternés qui lui demandaient plus d'informations. Selon lui, les femmes de la maison avaient trouvé le corps le matin et l'avaient informé de la mort de son père. Elles disaient qu'Abū Marwān avait été attaqué par un intrus pendant la nuit alors qu'il était seul dans sa chambre. Le savant vivait en effet dans la partie principale de la maison, séparé de sa famille, avec pour seul compagnon un adolescent nommé 'Abd al-Raḥmān, dont on ignorait qui était la mère et qu'il considérait comme son fils. Abū Marwān s'était ainsi isolé du reste de la famille qui comptait, outre le fils aîné, Ziyādat Allāh, sa femme et leurs enfants, la mère de Ziyādat Allāh et ses deux filles. Il les avait tous exilés dans la partie arrière de la maison, séparé de la partie principale par plusieurs portes.

Étant donné les circonstances, les gens voulaient voir le lieu du cambriolage, mais Ziyādat Allāh ne pouvait montrer aucune trace d'une incursion dans la maison. L'incohérence de ses explications

\footnotetext{
${ }^{4}$ Ibn Bassām, al-Dahīra fì mahāsin ahl al-ğazīra, ed. 'Abbās, I., Beyrouth, Dār al-Taqāfa, 1399/1979, 8 vols. (=(DY)), I, 537.

5 Sur la fortification de la partie Est, voir Müler, op. cit., $41 \mathrm{sq}$.

${ }^{6}$ Cf. Müller, op. cit., 321, annotation 57, et Makkī, M., «Usrat banī '1-Tubnī al-Qurțbiyyīn wa-mașra' Abī Marwān al-Ṭubnì, Mağallat Kulliyat al-adäb wal-tarbiya, 2 (1975), que je n'ai pas pu voir.
} 
laissait les auditeurs insatisfaits. De plus, il éveilla la suspicion de son auditoire en posant publiquement des questions trop précises sur les circonstances du meurtre, circonstances qu'il ne pouvait connaitre à ce moment-là, à moins d'avoir eu des informations de première main sur le meurtre.

Pendant l'audience que lui accorda le prince Ibn Ğahwar, le comportement du fils en deuil s'avéra inquiétant. Le prince était frappé par un décalage entre les lamentations de deuil à haute voix et l'expression rigide qu'il percevait dans l'œil du fils, qui manifestait plus de sérénité que de tristesse (littéralement: «[son] oeil sans mouvement reflétait la sérénité: 'ayn ğamūd tadullu 'alā sahwih»). En plus, Ibn Ğahwar avait du mal à croire qu'un intrus inconnu aurait assassiné ce savant innocent d'une façon si atroce. Il chargea donc son chef de police, le «préfet de la ville» (sạhib al-madina) Muhammad b. Hišām Ibn al-Muṣhafí le petit-fils ${ }^{7}$, de mener l'enquête sur cette affaire ${ }^{8}$.

L'enquête policière sur le lieu du crime nous paraît aujourd'hui être une procédure de routine. Pourtant, cela n'était pas le cas à Cordoue au XIème siècle, surtout si le mort avait été trouvé dans un lieu privé, par exemple à l'intérieur d'une maison. En cas d'homicide accidentel ou intentionnel (qatl hața' ou qatl 'amd), le droit prévoyait que des «mandataires du sang» (walī/wulät al-dam) issus de la famille de la victime réclament la peine de vengeance, le talion, ou le paiement du prix du sang par l'auteur ou du groupe des «contributes solidaires» ('aqīla). C'était le mandataire de sang qui produisait les témoins nécessaires, la charge de procureur officiel n'existant pas ${ }^{9}$.

${ }^{7}$ Abū Bakr Muhammad b. Hišām b. Muhammad b. 'Uțmān al-Qaysī (mort en 481/1088-9), était connu comme Ibn al-Muṣhafi al-hafid parce qu'il était le petit-fils du frère du Grand-vizir Ğa'far b. 'Uțmān al-Musḥ̣afí, cf. Ibn Sahl, Watāa'iq fì aḥkām

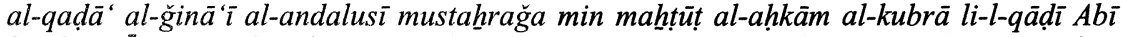

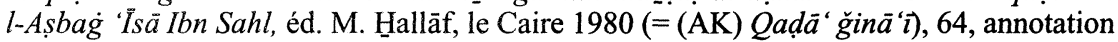
222. Cf. aussi Meouak, M., Pouvoir souverain, administration centrale et élites politiques dans l'Espagne umayyade (II'-IVe/VIII $-X^{e}$ siècles), Helsinki, 1999, 190, ainsi que 181-90, pour d'autres membres de la famille. Le même șâhib al-madina Muhammad b. Hišăm mena une autre enquête policière en $459 / 1067$, cf. Müller, op. cit., 311 sq. Sur les compétences policières de cette haute charge dans l'état omeyyade cf. ibid., 109-17 et index.

${ }^{8}$ Ibn Hayyān, cité dans (DY) I, 536.

9 Voir par exemple le cas de Rahīma Ibn Šuhayd, tuée à Cordoue en 462/1070 dans la maison par son mari, où le grand-père de la victime présente les témoins en tant que mandataire de sang, cf. Müller, op. cit., 316. 
Nous avons donc toutes les raisons de penser que le fait d'envoyer le chef de la police à la maison de la victime ne correspondait pas à une procédure de routine, mais venait du fait que le prince Ibn Ğahwar avait conçu des soupçons (tuhma/pl. tuham) envers Ziyādat Allāh, le fils aîné de la victime qui, dans des circonstances différentes, aurait été le mandataire de sang prioritaire pour son père. Il était dans la logique légale que le șâhib al-madina fût chargé de remplacer (tawkī) le suspect dans le rôle de «mandataire du sang» contre le meurtrier inconnu ${ }^{10}$. L'homicide ne demeurait donc plus une affaire privée mais devenait un procès engagé par un agent de l'État dans l'intérêt public.

Le chef de la police mena donc l'enquête. Il inspecta le lieu de crime mais il ne s'arrêta pas là. De plus, il tint compte de ce que les gens lui dirent et des doutes qu'ils exprimaient (qui n'avaient pas pu voir de traces d'une intrusion) et c'est pourquoi il interrogea les habitants de la maison ${ }^{11}$. L'inspection du lieu révéla une scène d'horreur: la victime baignait dans son sang sur le lit avec une soixantaine de coups de couteau dans l'artère $\mathrm{du}$ cou et dans la poitrine. L'interrogatoire du fils cadet 'Abd al-Raḥmān, à son tour, révéla des choses incroyables: l'adolescent, qui était atteint d'une maladie étrange qui affectait son esprit et son corps, déclara avoir vu la scène du meurtre. Selon lui, la femme d'Abū Marwān, c'est à dire l'esclavemère de Ziyādat Allāh, assistée de ses deux filles, aurait poignardé à mort le savant avec le couteau avec lequel il taillait les plumes. Le témoin ne mentionnait aucune participation active du fils Ziyādat Allāh au meurtre ${ }^{12}$. A la suite de cette révélation incroyable, le chef de la police leva la protection accordée aux femmes de la victime, et saisit par la force la mère de Ziyādat Allāh pour la frapper. Elle évita ensuite la torture par un aveu en faisant une description des circonstances de l'assassinat. Les inculpés furent donc emprisonnés ${ }^{13}$. Au lendemain du meurtre, on enterra Abū Marwān al-Ṭubnī; une foule impressionnante assista à la cérémonie en poussant des cris de vengeance et en demandant la punition pour les meurtriers. Dans ce contexte, Ibn Ḥayyān fait allusion à la haute réputation scientifique et humaine du

${ }^{10}$ Cf. (DY) I, 538.

11 Cf. ibid.

${ }^{12}$ Cf. ibid

13 La forme masculin pluriel «suğinū» nous fait penser qu'on a incarcéré au moins un homme, Ziyādat Allāh, avec des femmes (ibid.). 
savant assassiné ${ }^{14}$. Suivant les coutumes, Ziyādat Allāh, le fils, aurait dû être autorisé à conduire la prière pour le mort en vêtements de deuil blancs, mais il en fut empêché par ordre d'Ibn Ğahwar. Ce fut donc l'ami du souverain, le cousin au deuxième degré de la victime, le vizir Abū Bakr Ibrāhīm b. Yaḥyā al-Ṭubnī, qui conduisit cette prière ${ }^{15}$.

Jusqu'ici, notre analyse de l'assassinat d'al-Ṭunī suit les grandes lignes du récit donné par le célèbre chroniquer Ibn Hayyān qui s'avère être un observateur fiable des événements dans sa ville natale de Cordoue jusqu'à sa mort en 469/1076. Son récit du «cas Tubnī» porte sur les circonstances de vie et la motivation des participants, mais il n'oublie pas de mentionner les aspects juridiques qui déterminaient le destin des inculpés ${ }^{16}$. C'est Ibn Hayyān qui rapporte, de la façon la plus détaillée, les événements qui, après le décès d'al-Ṭubnī, avaient éveillé le soupçon contre le fils Ziyādat Allāh. Ibn Hayyān n'étant pas juriste lui-méme, on peut se méfier de sa présentation des faits juridiques. Pourtant, il était l'historien de la cour et probablement le mieux placé pour avoir accès aux dossiers administratifs. Son récit est donc bien informé et représente d'une certaine façon la «version publiée» des événements.

De plus, il existe une source d'un type différent qui met en valeur d'autres aspects du cas Tubnī: il s'agit du recueil des cas juridiques réunis par Ibn Sahl (mort 486/1093), al-Ahkãm al-kubrā. Nous avons toutes les raisons de penser que son auteur, Ibn Sahl, travaillait pendant les événements, de 457/1065 à 459/1067, comme greffier ( $k a \bar{t} i b$ ) chez un haut fonctionnaire d'état cordouan, le préfet de police et du marché (șăhib al-šurța wa-l-sūq) Muhammad b. al-Layt Ibn Harī̌s ${ }^{17}$. De plus, il était en contact personnel avec les juristes concernés par l'affaire. En conséquence, Ibn Sahl connaissait parfaitement le dossier d'al-Tubnī. Sa documentation juridique se présente sous la forme d'un récit du cas jusqu'à la délibération concernant les peines à infliger aux inculpés par le chef de la police dans le conseil judiciaire $(\check{s} u \overline{r a})$. Ce récit fut suivi d'un résumé des réponses faites par les trois

${ }^{14}$ Cf. (DY) I, 538.

${ }^{15}$ Cf. (IB(C)) I, n. ${ }^{\circ} 772,345$, sa biographie se trouve (IB(C)) I, n. $.^{\circ} 213,96$ sq.

16 Ibn Hayyān, cité dans (DY) I, 536-540, sous une forme «abrégée». D'autres citations courtes d'Ibn Hayyān se trouvent dans Ibn Sa'īd, al-Mugrib fí hulà l-Magrib, ed. Dayf, Š., El Cairo, 1953-55 (= (IS)), I, n. ${ }^{\circ} 25,92$, et (IB(C)), n. ${ }^{\circ} 772,345$.

17 Cf. Müller, op. cit., 3 sq., annotation 23. 
juristes Ibn 'Attāb, Ibn al-Qațtān et Ibn Mālik ${ }^{18}$. Notons la technicité du langage au sein du discours juridique, qui permet la discussion du cas considéré sans prendre en compte des attentes de vengeance. Les faits légaux exprimés dans les réponses juridiques sont encore plus différenciés que chez Ibn Hayyān. Pourtant, le récit du cas par Ibn Sahl n'est pas strictement chronologique: dans le récit d'Ibn Sahl en effet, l'enterrement du mort est mentionné avant le récit de l'enquête sur les lieux du crime, ce qui n'est logiquement pas possible puisque nous savons que la police avait vu le mort sur son lit. Pourtant, cet ordre des choses (enterrement - enquête) s'impose si on veut faire état de l'intégralité de données dans le compte-rendu de l'enquête, et dans la mesure où l'enquête policière s'est prolongée bien après l'enterrement. Pour ce qui est des résultats de l'enquête policière menée sur place et des déclarations des membres de la maison, le récit d'Ibn Sahl est plus détaillé que celui d'Ibn Hayyān.

Une fois lancée, l'enquête policière s'étendit à la totalité de la maison du savant décédé et ne s'arrêta pas devant les appartements privés des femmes. On ne trouva pas traces d'une intrusion dans la maison ni d'une fuite hors de la maison, mais on découvrit les vêtements de la victime cachés dans des coins de la maison ainsi que des pantalons féminins avec des traces de sang. Le «couteau de plume» appartenant au savant et avec lequel il avait probablement été tué, avait été jeté dans une autre chambre de la maison. Le policier fit parler les femmes. L'une d'entre elles avoua: «une autre femme a tué Abū Marwān, et nous autres l'avons aidé à le faire, et il le méritait depuis longtemps.» ${ }^{19}$ Selon le récit de la source juridique, le fils cadet qui avait le corps frappé d'incapacité «comme s'il était bousculé par le vent» ${ }^{20}$ aurait renoncé à son premier témoignage selon lequel il y avait eu un intrus nocturne et dit «les femmes l'ont tué pendant que son frère aîné restait derrière la porte» ${ }^{21}$. Ibn Hayyān ne parle pas du refus du fils cadet, pour lui ce n'est évidemment pas important. Mais Ibn Sahl y revient dans un court commentaire sur les avis des juristes Ibn al-Qatțān et Ibn Mālik, ${ }^{22}$ et il faut donc croire que le refus du fils

18 ((AK) Qaḍā' ǧinā'î), 63-68, repris, légèrement abrégé, par al-Wanšarīsĩ, al-Mi yār al-mu 'rib, ed. Hağğì, M., Rabat et Beyrouth, 1981, 13 vols. (= (MMW)) II, 324-26.

19 ((AK) Qadă' ğinā'î), 64 (MMW) II, 324.

20 "qad darabat-hu rịḥ» ((AK) Qaḍa'‘ ğinā' $\vec{\imath}), 64$ (MMW) II, 324.

21 Ibid.

22 ((AK) Qạ̣ā' ğinā' $\mathbf{\imath}), 68$, en haut de page. 
cadet avait des conséquences juridiques. D'autre part, on voit que l'ordre chronologique des choses est d'une importance secondaire dans le récit d'Ibn Sahl, mais les faits légaux, en revanche, sont exposés par lui avec plus de soin que chez Ibn Hayyān. Ibn Sahl explique surtout les données sur lesquelles les juristes ont basé leurs avis juridiques. Cela nous amène à penser que les faits exposés par lui sont devenus les «faits officiellement acceptés» du crime. Il ne reste qu'à constater que le texte juridique ne parle pas de menaces ni de torture afin d'obtenir un aveu. Vu les argumentations des juristes et la libération des inculpés, on peut supposer qu'il n'existait que ce seul aveu de la mère de Ziyādat Allāh, mentionné plus haut (on reviendra sur cette question).

Voici comment se déroula le procès: une première séance devant le qā $q \grave{\imath}$ régla la succession du savant assassiné. On constata légalement la mort d'Abū Marwän al-Tubnī ainsi que l'identité des ayant droits à l'héritage (warata). On décida également d'exclure définitivement Ziyādat Allāh des «mandataires du sang» (wulāt al-dam), cette fonction étant dorénavant attribuée au fils cadet 'Abd al-Raḥmān et aux deux neveux de la victime ${ }^{23}$ - le chef de la police (sāhib al-madi$n a)$ n'assuma ce rôle que le temps de l'investigation policière. Il faut noter que les noms des héritiers ne sont pas indiqués dans nos sources: le fait qu'aucune des femmes ne soit mentionnée parmi les wulāt al-dam n'exclut pas qu'elles aient hérité parce que le droit mālekite interdit aux femmes d'être wali al-dam dans des meurtres «intentionnels» (qatl 'amd), c'est à dire des meurtres par n'importe quel instrument) ${ }^{24}$. Le récit de l'historien selon lequel 'Abd al-Raḥmān, l'enfant (walad) du défunt, traité légalement en qualité de fils de la victime, «n'avait pas de mère» ${ }^{25}$ attire l'attention sur la parenté de l'enfant. Qu'on ignore le nom de sa mère signifiait qu' Abd al-Rahmān n'était

23 ((AK) Qaḍā' ǧinā' $\overrightarrow{)}) 64$ sq.: wa-tubita mawtu-hu wa-wirātatu-hu wa-an ibnay ahi-hi ahaqqu al-näs bil-qiyām bi-dami-hi ma 'a ibni-hi al-da 'îf. L'attestation (țubüt) des

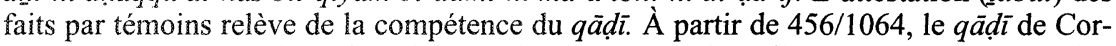
doue était Muhammad b. Ahmad b. Mahlad Ibn Baqī, cf. Müller, op. cit., 422: il présida plusieurs procès, ibid., 212-24, 218 sq., 228-34, 236-45.

${ }^{24}$ Cf. Ibn 'Abd al-Barr, Abū 'Umar Yūsuf b. 'Abd Allāh, al-Kāfífí figh ahl al-madina al-mãlikī, Beyrouth, Dār al-kutub al-'ilmiyya, 1987, 604. Ce manuel de droit mālikite andalou du XIème siècle nous permet de mieux comprendre les normes légales en vigueur à l'époque. Donc, tous les héritiers ne sont pas des wulāt al-dam, contrairement à

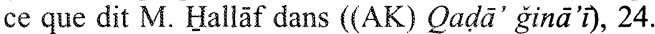

25 (DY) I, 537. 
pas né d'une épouse connue à Cordoue et que sa filiation n'avait été établie qu'en ligne patrilinéaire. Rappelons à ce propos que le savant avait longuement voyagé en Orient avant de revenir à sa ville natale et qu'il aurait pu avoir des enfants dans un pays d'Orient ou avec une femme, esclave ou non, dont il n'avait pas révélé l'identité. Rappelons encore que la reconnaissance de paternité (istilhạq) par le père ou par ses parents proches créait les liens légaux, même si une telle reconnaissance concernait une personne autre que l'enfant biologique. La seule différence légale entre un enfant biologique et un autre enfant accepté par reconnaissance de paternité présumée réside dans le fait que ce dernier n'hérite pas, dès lors que la fiction de la filiation présumée est reconnue comme fictive par le tribunal ${ }^{26}$. Cela dit, dans la mesure où nous ne possédons pas la liste de les héritiers d'Abū Marwān, nous ne pouvons pas savoir de façon certaine si 'Abd al-Rahmān était bien son fils biologique. Nous reviendrons sur la question de l'héritage après avoir fait état du procès criminel. 'Abd al-Raḥmān, le fils désigné comme «mandataire du sang», ne fut donc plus soupçonné d'avoir tué son père avec qui il vivait, grâce aux bonnes relations personnelles qu'il avait eues avec celui-ci avant sa mort, et grâce à la reconstitution du crime sur la base de l'investigation policière.

Le châtiment des auteurs du crime n'était pourtant pas l'affaire du $q \bar{a} d \bar{d}$. C'est le săhịib al-madina qui convoqua le conseil de juristes de la ville (šūrā) pour délibérer sur le sort des inculpés ${ }^{27}$. Ibn Hayyān explique: «le sultan a demandé de retarder l'exécution de la peine de hadd contre eux à cause des doutes émergents» ${ }^{28}$. Le délit susceptible d'être sanctionné par une punition dite hadd n'est pas spécifié dans la source. Etant donné le nombre limité des types de meurtres soumis à la peine de hadd en droit mālikite, on peut déduire de la phrase d'Ibn Hayyān que nous venons de citer que l'inculpation des membres de la famille portait au départ probablement sur le crime de muhāraba, «meurtre par cupidité». Au contraire d'un meurtre «normal», le meur-

${ }^{26}$ Cf. Landau-Tasseron, E., «Adoption, acknowledgment of paternity and false genealogical claims in Arabian and Islamic societies", $B S O A S$, 66 (2003), 187 sq. Je remercie Maribel Fierro de m'avoir indiqué cet article. Cependant, il demeure que les implications légales d'une reconnaissance de paternité basée sur une fiction demandent à être étudiés plus à fond.

${ }^{27}$ ((AK) Qad̄a' ğinā' 't), 65.

28 (DY) I, 539. 
tre par cupidité est considéré comme un délit de droit public (hadd). Dans ce cas, les condamnés sont exécutés par ordre du souverain sans que les parties civiles, les wulāt al-dam aient une décision à prendre ${ }^{29}$.

La discussion juridique dans le cas du meurtre d'al-Tubnī porte sur deux aspects légaux différents qui se rejoignent cependant au niveau des faits historiques: faut-il appliquer la peine de mort dans un cas de droit public (hadd) ou faut-il s'en abstenir pour cause de doutes? Si le hadd n'est pas appliqué, ce sont en général les parties civiles qui décident de la mort d'un inculpé: si une personne est soupçonnée d'un meurtre et qu'il y ait des indices qui l'accusent sans qu'il y ait de témoins légaux ( $\check{s} u h \bar{u} d)$ de fait, il est «suspect». Dans ce cas où il existe des indices inculpants (lawt), les mandataires du sang peuvent prêter le «serment cinquantenaire» (qasāma) pour inculper l'accusé du meurtre en question. Celui-ci serait à son tour soumis à la peine du mort, le talion (qiṣass), si la famille du victime ne lui a pas accordé son pardon contre le paiement du «prix du sang» (diya). Voilà donc pourquoi il est fondamentalement important de connaître qui devient «mandataire du sang» pour les inculpés à partir du moment où la punition par droit public (le hadd) n'est pas appliquée ${ }^{30}$. Les droits des parties privées dans un cas de meurtre sont réglés au chapitre des délits de sang $(\operatorname{dim} \bar{a})$. Il faut souligner le fait qu'un procès de droit public (hadd) nécessite d'autres formes de preuves légales beaucoup plus restreintes que celles exigées dans un procès de talion (qișās).

En plus des deux champs judiciaires concernés, la reconstitution du meurtre d'al-Ṭubnī sur le plan juridique est compliquée par l'état des sources. Tout d'abord, Ibn Sahl ne reproduit pas l'intégralité des réponses $(f a t \bar{a} w \bar{a})$ mais choisit quelques points qui lui apparaissent comme décisifs. Cela ne permet la reconstruction de l'argumentation pour cha-

\footnotetext{
${ }^{29}$ Pour la définition de muhāraba cf. Ibn 'Abd al-Barr, Käfì, 583. L'autre crime meurtrier puni par hadd est le brigandage (qați ' al-țuruq) qui ne s'applique évidemment pas aux membres de la maison. Il existait une tendance générale chez les juristes à limiter l'application des peines de hadd pour raison de doute (šubha), cf. Schacht, J., An Introduction to Islamic Law, Oxford, 1964, 1982 $2^{5}, 176$ sq., et Johansen, B., «Eigentum, Familie und Obrigkeit im hanafitischen Strafrecht. Das Verhältnis der privaten Rechte zu den Forderungen der Allgemeinheit in hanafitischen Rechtskommentaren», Welt des Islams, XIX (1979), 1-73, en part. 44-46, 53.

30 Voir la polémique autour de la question: qui devient «mandataire de sang» dans un autre cas de meurtre, Hallaq, W., «Murder in Cordoba: Ijtihäd, Iftä and the Evolution of Substantive Law in Medieval Islam», Acta Orientalia, LV (1994), 55-83, part. p. 64.
} 
que auteur qu'à partir des réflexions d'Ibn Sahl qui, on va le voir, met en valeur l'opinion exprimée par son maître Ibn 'Attāb. A titre complémentaire, la deuxième source, le récit de l'historien Ibn Hayyān, cite également certains éléments des expertises juridiques. Mais ces éléments ne correspondent pas à ceux fournis par Ibn Sahl. C'est pourtant en comparant ces deux sources complémentaires que l'on arrive à bien mettre en lumière les éléments de la discussion juridique et de la prévention juridique tacite dont Ibn Sahl donne la preuve.

La seule fatwā dont Ibn Sahl fournit plusieurs éléments est celle de son maître, le juriste Ibn 'Attāb (m. 461/1069). Dans sa fatwā, Ibn 'Attāb met en évidence les doutes légaux ( $\check{s} u b h a)$ qui le mènent à ne pas demander une peine légale (hadd) pour les auteurs du crime. Il relève l'ambiguïté de la déclaration d'une femme concernant une autre femme: «elle a tué [al-Tubnī] et nous toutes l'avons aidé». Selon lui, cette déclaration peut désigner soit une aide verbale, soit une aide factuelle, ce qui fait que l'aveu n'est pas considéré comme assuré de porter sur une aide factuelle ${ }^{31}$.

Il faut savoir que, selon la Loi sacrée qui détermine l'application des peines légales, un aveu (iqrār) n'est légalement valable que pour la personne qui le fait: dans notre cas, le fait d'avouer avoir assisté au meurtre ne concerne donc pas les autres femmes. Et d'autre part, l'autre forme de la preuve légale en droit public (hudüd), est le témoignage (šahāda), or la possibilité de témoigner ne s'appliquait pas aux femmes en cas d'homicide ${ }^{32}$. La déclaration faite par la mère de Ziyādat Allāh concernant le meurtre n'aurait donc pas été considérée par Ibn 'Attāb comme une preuve juridique incontestable. La deuxième raison de douter consiste, selon Ibn 'Attāb, dans le fait que cette déclaration ne fut faite qu'aprés l'interrogatoire par la police, un interrogatoire dont les femmes de la maison avaient peur ${ }^{33}$. Dans cette perspective, la dernière partie de la phrase peut être interprétée comme un indice de la coercition ou même des tortures qui faisaient partie des méthodes d'investigation policière. Pour les juristes à l'époque classique, ces méthodes entachent la validité d'un aveu légal dans le procès de droit public concernant la punition de hadd ${ }^{34}$. La conclusion légale

31 ((AK) Qaḍa ’ ğinā' $\mathbf{)}), 65$ sq.; (MMW) II, 325.

32 Cf. Ibn 'Abd al-Barr, Käfi, 469.

33 ((AK) Qad̄à' ğinā' $\vec{\imath}), 65$ sq.; (MMW) II, 325

34 Cf. Johansen, B., «La découverte des choses qui parlent. La légalisation de la torture judiciaire en droit musulman (XIIIè - XIVè siècles)», Enquête, 7 (1998), 175-202, 
suit donc le raisonnement du droit procédural dans le domaine du hadd, selon lequel les autorités publiques n'avaient pas suffisamment de preuves pour établir une conviction ayant pour conséquence la peine de mort pour le délit de hadd en question, le meurtre pour motif de cupidité (muhāraba). Pourtant, à aucun moment Ibn 'Attāb ne met en doute la parole de la femme dans sa déposition.

Selon Ibn Hayyān, le sultan demanda à retarder l'exécution de la peine de hadd contre les inculpés à cause des doutes émergents ${ }^{35}$. Nous ne savons pas si les autres juristes n'étaient pas d'accord, parce que les éléments de leurs opinions fournis par les sources portaient sur un autre aspect de l'affaire: celui de l'aptitude du fils cadet à agir en tant que «mandataire de sang» pour son père assassiné.

Les juristes Ibn al-Qațtān et Ibn Mālik ne sont cités par Ibn Sahl qu'en deux mots sur leur opinion différente de celle d'Ibn 'Attāb: tous deux considérant en effet qu'il est possible d'agréer le «fils frappé d'incapacité ( $d a$ ' $i f f) »$ comme mandataire de sang. Selon Ibn Sahl en revanche, cet avis est «sujet à caution (fi-hi nazar)» dans la mesure où le fils «frappé d'incapacité» avait dans un premier temps dit que le crime avait été perpétré par un intrus. Quant à Ibn 'Attāb, Ibn Sahl ne mentionne pas son opinion sur ce point ${ }^{36}$.

De son côté, le chroniqueur Ibn Hayyān nous informe qu'il y avait toute une histoire liée avec cet aspect de l'affaire: le juriste Ibn al-Qaț̣ān aurait exigé de mieux connaître l'état de santé du fils malade avant de donner son avis juridique: Si la maladie était corporelle et ne touchait pas l'esprit ('aql), s'il était comme capable de discernement (mumayyiz), il serait «mandataire de sang», sans que les deux neveux de la victime et les fils de son oncle paternel puissent décider à sa place. Cette question résolue, on donnerait au juge un avis juridique (fatwā) à sa demande ${ }^{37}$. Par conséquent, le fait qu'Ibn Sahl ait écrit que les conseillers Ibn al-Qațtān et Ibn Mālik s'étaient prononcés pour le droit du fils à être mandataire du sang ${ }^{38}$ ne peut que donner une certitude: un examen médical du fils malade avait eu lieu et on considérait sa

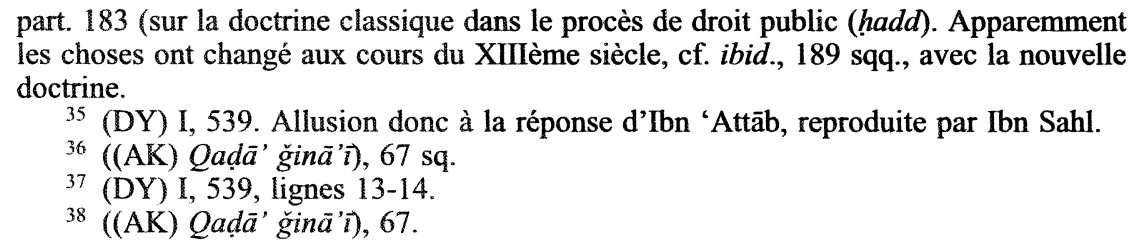


maladie comme physique et non pas mentale ${ }^{39}$. Ibn 'Attāb déniait cependant, selon Ibn Hayyān, son droit au fils malade, et les juristes n'étaient pas d'accord sur ce point. Pourtant, les conseillers juridiques étaient tous d'accord sur le fait que les inculpés soient sévèrement battus et, par la suite, incarcérés pendant une longue période ${ }^{40}$.

Le résultat du procès peut cependant nous paraître étonnant. Car nos deux sources principales font état de la décision du sultan Ibn Ğahwar en faveur de l'avis d'Ibn 'Attāb. A la suite de cette décision, les trois inculpés, c'est à dire le fils aîné, sa mère et l'autre esclave-mère (la femme de Ziyādat Allāh) avaient, afin d'être finalement libérés, prêté une deuxième forme de "serment cinquantenaire», appelé lui aussi qasāma, par lequel chacun jurait n'avoir pas participé au meurtre ${ }^{41}$. Comment en était-on arrivé à cette conclusion judiciaire qui était opposée aux attentes du public à l'époque de l'enterrement de la victime? Constatons encore une fois que la dispute des juristes telle qu'elle est visible dans les sources - ne portait pas sur l'application d'une hadd-punition: les juristes étaient probablement d'accord sur les doutes légaux qui empêchent l'exécution par crime de hadd. Pourtant, leur dispute pour ou contre l'application du talion avec la possibilité d'exécuter les inculpés était décisive, si les mandataires de sang le voulaient. Selon le droit mālikite de l'époque, l'application de talion nécessitait des indices bien fondés (lawt $)$ contre le, ou les inculpés. Dans ce cas seulement, deux mandataires de sang peuvent prêter serment pour inculper celui qui est accusé de meurtre. Celui-ci serait à son tour soumis à la peine de mort, s'il n'était pas pardonné par la famille de la victime contre le paiement du prix du sang ${ }^{42}$. La solution finalement adoptée dans le cas d'al-Ṭubnī: le serment d'innocence prêté par les inculpés, nous conduit à penser que le juriste qui est à l'origine de cela, Ibn 'Attāb, avait exclut que les mandataires de sang puissent inculper les auteurs sur la base des indices inculpants $(\text { lawt })^{43}$.

39 À la condition qu'Ibn Sahl n'ait pas abrégé l'opinion desdits juristes en confondant la condition (s'il est mumayyiz il devient accusateur du sang) avec le fait accompli.

40 (DY) I, 539, l'expression «bi-tațil siğni-him» porte sur le fils aîné et les femmes. Un tel accord entre les juristes n'est pas mentionné par Ibn Sahl.

${ }^{41}$ ((AK) Qada $\bar{a}^{\prime}$ ğinā' $\left.\mathbf{\imath}\right), 68$, et (DY) I, 539 sq.

42 Cf. Müller, C., op. cit., 169 sq.

${ }^{43}$ Les sources n'indiquent pas que les mandataires de sang ont accepté le paiement du prix du sang, ce qui pourrait constituer une autre possibilité d'éviter le talion, voir le cas d'Ibn Šuhayd, Müller, op. cit., 318 sq. 
Une explication possible est donc que la déclaration du fils frappé d'incapacité $\left(d a^{\prime} i \bar{f}\right)$ n'était pas considérée comme suffisante pour créer des indices inculpants (lawt) contre le fils aîné et les femmes. Selon le droit, un lawt contre l'inculpé de meurtre est basé sur un témoignage d'une seule personne à condition qu'i s'agisse d'un homme, qu'il soit musulman, libre et majeur ${ }^{44}$. Le fils malade ne fut pourtant jamais appelé «mineur», et le fait de ne pas avoir la capacité de témoigner ('adāla) n'exclut pas non plus que le témoin devienne mandataire de sang ${ }^{45}$. On ne voit donc pas bien pourquoi la déclaration d' Abd al-Raḥmān n'avait pas valeur d'indices inculpants. Et même si 'Abd al-Raḥmān n'était pas considéré comme mandataire de sang, on aurait pu trouver d'autres membres du clan al-Ṭubnī qui prètaient serment pour engager le talion ${ }^{46}$. Des cas semblables existent.

Ici, le fait qu'aucun membre de la vaste famille d'al-Ṭubnì à Cordoue ne soit connu pour avoir prêté le «serment cinquantenaire» et revendiqué le talion, ni par son refus de le faire, ${ }^{47}$ me conduit à penser qu'Ibn 'Attāb avait refusé la possibilité de revendiquer le talion malgré le fait qu'il voyait les indices graves à retenir contre les inculpés.

Cela dit, les éléments connus de l'argumentation d'Ibn 'Attāb vont dans une autre direction. Il dit que (tous) les habitants de la maison ne doivent pas être tués mais incarcérés longtemps après avoir prêté le "serment cinquantenaire» de ne pas avoir tué, ni participé au meurtre. Son argument est basé sur un cas similaire, décidé par le juge cordouan Abū Bakr Ibn Zarb, mais qui n'est pas conforme à la pratique judiciaire ('amal) ${ }^{48}$. Je n'ai pas pu identifier ce cas dont Ibn

${ }^{44}$ Un mineur ne pouvait pas décider de la mise à mort par qișās, cf. Ibn 'Abd al-Barr, $K \bar{a} f i, 592,600$.

${ }^{45} \mathrm{Cf}$. Ibn 'Abd al-Barr, Käfi, 601.

46 Sur le dispute concernant le cercle des parents qui peuvent devenir mandataires de sang, voir Hallaq, W., Murder in Cordoba, 64, avec l'argumentation d'Ibn Rušd (le grand-père du philosophe) selon laquelle seulement les fils du victime pourront exiger le talion.

47 Même le fait qu'un mandataire de sang ait refusé le serment est mentionné comme un fait important dans autres cas, voir par exemple pour Ibn Šuhayd ((AK) Qadẹa' ğinä' $\mathbf{\imath}), 71$.

${ }^{48}$ ((AK) Qaḍa ' ğinā ‘ î), 65. Pour 'amal dans le contexte d'al-Andalus cf. Chalmeta, P., "Acerca del 'amal en al-Andalus: algunos casos concretos», Anuario de Historia del Derecho Español, 57 (1987), 339-64; Carmona, A., «Las diferencias entre la jurisprudencia andalusí y el resto de la escuela de Mālik: el texto atribuido a Abū Ishāāq al-Garnātīi, Al-Qanțara, XIX (1998), 67-102. Serrano, D., «La prática legal ('amal) en al-Andalus durante los siglos X-XII, a través de los Madāāhib al-hukkām fi nawãzil al-aḥkām de Muhammad Ibn 'Iyāḍı, Qurțuba I (1996), 171-92. Au XIème siècle, 'amal n'avait pourtant pas en- 
Sahl dit qu'il l'avait cité plus haut dans un contexte différent, celui des rapports sexuels ( 'išra) ${ }^{49}$. L'essentiel est pourtant que, selon Ibn Sahl, Ibn 'Attāb se serait opposé aux possibilités de «serment cinquantenaire» pour les mandataires de sang (même avec de lawt) dans le cas d'al-Tubnī. Et l'on ${ }^{50}$ cite dans ce contexte une opinion de Mālik dont la solution correspond exactement à celle qui fut acceptée par Ibn Ğahwar sur proposition d'Ibn 'Attāb: un homme meurt soudain pendant l'acte sexuel et la femme est soupçonnée. Malik conseille au mandataire de sang d'enquêter sur les circonstances de l'affaire, et si la femme n'est pas soupçonnée, elle ne devrait être ni incarcérée ni soumise aux menaces. Le disciple de Mālik, Ibn al-Qāsim, ajoute que s'il y a soupçon, elle sera incarcérée et si on ne trouve rien contre elle, elle pourrait prêter le «serment cinquantenaire» après une incarcération prolongée ${ }^{51}$.

Ce scénario légal expliquerait pourquoi le fils cadet d'al-Tubni, 'Abd al-Raḥmān, non inculpé par les indices de crime, ne prêtait pas le «serment cinquantenaire» malgré le fait qu'il faisait partie des habitants de la maison et n'était pas mandataire de sang. De plus, cela expliquerait pourquoi les mandataires de sang n'avaient pas eu la possibilité de demander le talion malgré les indices existant contre les inculpés. Au départ, Ibn 'Attāb avait exigé le serment et l'incarcération pour les deux fils, et pas seulement pour le fils aïné 52 .

La mention du cas d'un homme mort pendant l'acte sexuel, est-elle due à un hasard de l'argumentation juridique ou bien, en revanche, est-elle révélatrice de faits dans l'assassinat d'al-Tubnì qui autrement auraient été dissimulés par pudeur dans les sources? On peut en effet imaginer que le savant avait une relation homosexuelle avec l'adolescent 'Abd al-Raḥmān qu'il aurait adopté comme fils et avec qui il vivait dans la mème maison. De plus, le récit d'Ibn Ḥayyān sème le doute sur l'identité d'Abd al-Raḥmān «dont on con-

\footnotetext{
core la même signification doctrinale que dans le malikisme tardif, cf. maintenant Müller, C., «Sitte, Brauch und Gewohnheitsrecht im malikitischen fiqh» Kemper M. et Reinkowski M. (éds.), Rechtspluralismus in der Islamischen Welt. Gewohnheitsrecht zwischen Staat und Gesellschaft, Bamberg 2005, 17-38, part. 33-6.

49 ((AK) Qadä' ğinä' $\mathbf{i}), 66$, lignes 5-6.

50 Le texte n'indique pas dans une façon claire, s'il s'agit de la fatwā d'Ibn 'Attāb ou de l'explication d'Ibn Sahl, ibid., 67.

${ }^{51}$ ((AK) Qad̄̄' ğinā'ì), 67.

52 Ibid., 65, ligne 4, les fils en dual.
} 
naissait pas la mère». Mais on pourrait ajouter à cette hypothèse qu'une telle relation homosexuelle, vécue dans l'intimité d'une maison, aurait pu être indifférente au reste de la famille dans une société qui tolérait ce type de rapports sexuels, du moins dans la sphère privée ${ }^{53}$. Il existe trois indices suggérant que le meurtre ait eu lieu durant un tête-à-tête intime entre le savant et l'esclave la plus jeune de la maison. Tout d'abord, cela expliquerait comment les acteurs de crime pouvaient franchir les portes entre la partie de la maison qui leur était réservée et celle du vieux savant méprisant: ces portes étaient simplement ouvertes pour laisser passer l'esclave pendant la nuit. Ensuite, suivant l'aveu cité dans l'expertise d'Ibn 'Attāb, ce n'était pas la mère du fils aîné qui avait porté les coups de couteau, mais une autre femme. Si le vieux savant avait pu exercer son pouvoir sur une esclave féminine qui faisait partie de sa maison, il y avait de fortes chances qu'il s'agit de l'esclave la plus jeune, celle qui avait des enfants de Ziyādat Allāh. Un tel comportement expliquerait, bien sûr, la haine à mort du fils aîné contre son père. On sait que la relation sexuelle d'un homme avec les esclaves de son fils, ou avec des parentes de ses propres concubines, était formellement interdite par la loi qui la considérait comme une injustice (ta'ädin) ${ }^{54}$. L'interdiction absolue des actes sexuels avec ces personnes était d'autant plus sévère (ašadd al-tahrim) que le délit n'était pas sanctionné par une peine du droit public (hadd) ${ }^{55}$. Cela dit, un tel acte n'était pas considéré comme adultère $(z i n \bar{a})$ et la sanction qu'elle impliquait suivait les règles du droit civil sous la forme d'indemnisations. Si l'esclave du fils avec laquelle le père entretenait un rapport sexuel était l'esclave-mère de ce fils, l'indemnisation du fils était plus élevée que pour un esclave d'une autre catégorie: Dans ce cas, le père devait verser à son fils le valeur (qima) de l'esclave-mère au jour du coït (wat') «parce que le père avait privé le fils de son droit (lil-fasād allad̄i adhala-hu 'alā $a l-i b n) »$, et que, dans ce cas, l'esclave impliquée devait être libérée

53 Cf. Adang, C., «Love between Men in Tawq al-hamāma», dans Puente, C. de la (Ed.), Identidades Marginales (EOBA XIII), Madrid 2003, 111-41, part. 112 sq. et $138-41$.

${ }^{54}$ La norme juridique ne s'arrête pas à l'interdiction, contrairement à ce que peut faire croire l'article de Puente, $C$. de la, «Entre la esclavitud y la libertad: consecuencias legales de la manumisión según el derecho mālikì), Al-Qantara, 21 (2000), 344, voir infra, annotation 55 .

55 Pour l'argumentation juridique voir Sahnün, al-Mudawwana al-kubrāa, 6 vols., Le Caire, réimpr. Beyrouth, s. d., en part. II, 285, ligne 6. 
tout de suite ${ }^{56}$. On comprend bien qu'une telle «solution» n'ait pas plu au fils aîné, Ziyādat Allāh, qui aurait donc perdu, si sa concubine avait accédé à la liberté, ses propres droits sur elle.

La déclaration du fils cadet, selon laquelle sa femme, la mère de Ziyādat Allāh, et ses deux filles auraient attaqué le vieil homme, ${ }^{57}$ paraît à première vue contrarier l'hypothèse selon laquelle le vieil homme aurait eu des rapports avec l'esclave mère de son fils au moment du meurtre. On se demande néanmoins pourquoi la femme de Ziyādat Allāh eut à prêter serment si elle n'était également présente au lieu de crime. Reste également inexpliquée la raison pour laquelle les deux filles d'al-Tubnī ne sont pas mentionnées parmi ceux qui prêtèrent serment pour sortir de la prison. Enfin, troisième indice en faveur d'un crime passionnel soulignant ce rapport de dépendance entre le savant et des femmes esclaves vivant sous son toit: plusieurs sources parlent du «meurtre d'al-Tubnì par ses femmes (nis $\left.\bar{a}^{\prime} u-h u\right)^{58}$ ou ses esclaves (ğawärī-hi) ${ }^{59}$ en ignorant que l'une d'elles était également la femme du fils aîné. Il ne s'agit cependant que d'hypothèses. Nos sources sont complètement muettes sur la possibilité d'un assassinat pendant ou avant un acte sexuel.

Revenons aux faits historiques. Pendant la période qui a suivi le crime, il s'est tenu au moins trois séances des trois autorités judiciaires différentes: le $q \bar{a} d i$, le chef de la police (săhib al-madina), et le souverain.

Dans la première séance tenue par le $q \bar{a} d \bar{l}$, après l'information judiciaire, les questions du droit civil lié à la mort d'al-Tubnī furent réglées. De ce fait, le chef de la police (sāhibib al-madina), Ibn al-Mushafî, perdait les pouvoirs légaux qui vont avec le rôle de mandataire de sang et c'est le fils cadet de la victime, 'Abd al-Rahmān qui, avec deux des neveux de la victime, deviennent mandataires de sang (voir supra).

\footnotetext{
56 Sahnūn, Mudawwana, III, 320-1, part. 321 ligne 16. Cette procédure correspondait au cas d'un partenaire (šarik) qui avait acheté, avec le capital commun, une esclave pour l'acte sexuel, voir $i b i d$., III, 3211 . 1 et $i b i d ., \mathrm{V}, 73,11.1-2$. Le père qui avait une relation avec une esclave de son fils, autre que l'esclave-mère, lui payait sa valeur et devenait maître de l'esclave. Cependant, il devait respecter une période d'istibra $\bar{a}$ ' parce que l'acte sexuel initial était une transgression et que «le mélange de l'acte licite avec l'illicite est interdit»: ibid., III, 133.

57 (DY) I, 538.

58 Ibn Hayyān dans (DY) I, 537, ligne 5.

59 Ibn Sa'īd (IS) I, 92: ğawäri-hi qatalanna-hu.
} 
Deuxième séance: plus tard, les conseillers juridiques de la ville furent convoqués par le chef de la police, Ibn al-Mușhafî, pour émettre des fatwā-s (expertise juridique) concernant le sort à réserver aux membres de la maisonnée ${ }^{60}$. Le juriste Ibn 'Attāb, dans la fatwā qu'il avait émise, et qu'il avait probablement donnée à Ibn al-Muṣhafi, préconisait d'incarcérer tous les habitants adultes de la maison, le fils cadet 'Abd al-Raḥmān inclus. Ce juriste n'acceptait donc pas que 'Abd al-Raḥmān fût mandataire de sang de son père, bien que le conseiller Ibn al-Qatțān voulût faire examiner son état de santé mentale, et de ce fait les autres conseillers, Ibn al-Qatțān et Ibn Mālik, acceptaient le fils en qualité de mandataire de sang. Une chose n'est pas claire: la demande d'examen concernant la capacité mentale du fils cadet a-t-elle été discutée dans une autre séance que celle où ont été présentés les résultats de l'enquête, soit devant le qādī ou, ce qui serait le moins logique en termes de droit, devant le chef de police? En tout cas, la multiplication des séances et en conséquence des conseils juridiques expliquerait le fait que Ibn Sahl n'eût pas transmis les réponses juridiques intégralement, même pas celles de son maître Ibn 'Attāb.

Nous ne pouvons pas trancher la question de savoir si le refus d'Ibn 'Attāb d'accepter la peine du talion aprés le serment des mandataires de sang était fondé sur un manque d'indices inculpants (lawt) ou sur le cas d'une femme se trouvant en situation d'intimité avec un homme qui meurt. Cependant, nous devons bien constater que cette décision, qui allait contre la pratique légale ('amal) de la ville, ou plutôt les circonstances dans lesquelles le souverain les accepta, fit scandale. En effet, à Cordoue pendant la période des taïfas, c'est le souverain qui prenait personnellement la décision concernant les peines de mort et les exécutions, et non son chef de police, ni le $q \bar{a} \underline{d} \bar{\imath}^{61}$.

Troisième séance: voici comment se déroulèrent les événements à partir du moment où le souverain prit les choses en main: quand le souverain Ibn Ğahwar convoqua les conseillers juridiques (mušāwarūn) de la ville pour assister à la proclamation du jugement dans la mosquée Gānim, juste à côté de la maison d'Ibn 'Attāb, ils refusèrent de venir à cause de ce traitement de faveur: le choix du lieu,

${ }^{60} \mathrm{Cf}$ ((AK) Qadă 'ăinā' $\left.\mathbf{t}\right), 65$, ligne 3.

${ }^{61}$ Cf. Müller, op. cit., 332. 
tout près de la maison d'Ibn 'Attāb ${ }^{62}$. On ne força pas les conseillers absents à se présenter. Le souverain décida donc de tenir la séance à la mosquée avec les notables et les magistrats (hukkām), c'est à dire le $q \bar{a} d \bar{l}$ et les autres autorités judiciaires de la ville, le préfet du marché (sāhib al-šurța wāl-sūq) et le préfet de la ville (sāhib al-madina) ainsi que le juge des plaintes (șăhib al-mazālim) ${ }^{63}$. Ibn Ğahwar ordonna d'aller chercher les deux fils et les deux neveux de la victime. Malgré le rôle que les femmes avaient joué dans l'affaire, leur présence dans cette séance publique n'est pas attestée. On lut publiquement les expertises juridiques, la šürā, et le souverain décida d'accepter la réponse d'Ibn 'Attāb. Un jugement correspondant à cet avis fut exécuté.

À la suite de cette décision, on l'a déjà dit, le fils aîné, sa mère et sa femme, prêtèrent chacun le «serment cinquantenaire» dans un endroit appelé «point de la justice» (maqta 'al-haqq) dans la Grande Mosquée et jurèrent «ne l'avoir pas tué, ni terrassé (malaka 'alay-hi), ni avoir participé à l'affaire» ${ }^{64}$. Ainsi échappèrent-ils à la peine de mort. En revanche, ils savaient qu'un parjure serait puni, selon la conviction religieuse, deux fois dans le monde futur. Le fils aîné, Ziyādat Allāh, resta longtemps en prison et, après sa libération, les notables (nās) évitaient sa compagnie car il était toujours soupçonné d'avoir participé à l'assassinat de son père ${ }^{65}$. En effet, en dépit d'un témoignage portant sur sa participation directe, les gens le considéraient comme l'instigateur (mudabbir) du meurtre ${ }^{66}$. On mentionna cependant son nom en qualité de transmetteur d'un vers de son père dans l'anthologie biographique par Ibn Pascual (Baškuwal), ${ }^{67}$ ce qui pourrait signifier qu'il avait des contacts avec les savants de sa génération.

Le sort des femmes inculpées n'est en revanche pas mentionné dans les sources. Le récit d'Ibn Hayyān donne l'impression que le

62 ((AK) Qadă' ğinā' $), 68$. Inutile de spéculer sur l'emplacement de cette mosquée en rapport avec le lieu de crime d'al-Tubnì dans l'Ajarquía, avec Mazzoli-Guintard, C., Vivre à Cordoue au Moyen Age, Rennes, 2003, 97, contre Pinilla Melguizo, R., «Jurisprudencia y ciudad. Notas sobre toponimia y urbanismo en la Córdoba altomedieval extraídas de al-Ahkām al-kubrà d'Ibn Sahl (siglo XI)», Martin, J.C. et al., Las ciudades históricas. Patrimonio y sociabilidad, Córdoba, 2000, 559-574, ici 569.

${ }^{63}$ Cf. Müller, op. cit., chapitre 3, pour ces magistrats en Cordoue.

${ }^{64}$ ((AK) Qadă ' ğinā' $)$ ), 65 et 68 (avec la variante «lā mäla' 'alay-hì). Le trois mots "lā malaka 'alay-hi» (de ne l'avoir pas terrassé) manquent dans un serment similaire qu'on avait exigé à Fuṭays, soupçonné du meurtre de sa femme Rahìma, cf. ibid., 71 .

65 Ibn Hayyān dans (DY) I, 540.

${ }^{66}$ Cf. ibid., 537. 
serment dans la Grande Mosquée précéda une longue incarcération. Dans ce cas, on peut dire que si les inculpés n'avaient pas prêté serment, ils ne seraient pas sortis de prison. Mais les sources ne sont pas claires sur ce point: d'autre part, on voit que les inculpés avaient évité le talion avec, en conséquence, leur exécution en prêtant serment avant même leur séjour prolongé en prison.

Impossible de dire si la police aurait fouillé la maison d'al-Ṭubnì, ce qui eut pour résultat le fait que la famille se trouva accusée, sans le soupçon initial qui pesait sur le fils aîné Ziyādat Allāh à la suite de l'audience que lui accorda le prince Ibn Ğahwar. Mais une fois commencée, l'enquête fut menée jusqu'au bout, y compris dans l'espace privé de la maison. Les cris publics de vengeance n'avaient pas eu raison de la prudence des juristes, ni de celle du souverain, et les inculpés échappèrent à la peine de mort. Pourtant, les résultats de l'enquête policière changèrent radicalement les aspects juridiques du meurtre: Si la version initiale du meurtre par un intrus avait été admise, tous les «contributes solidaires» ('aqĩla) du lieu dont on trouva le corps auraient payé le prix du sang après prestation collective du «serment cinquantenaire» de n'avoir pas tué la victime. Dans ce cas de figure, on constate de façon certaine que les deux fils, mais non pas les deux femmes esclaves, auraient participé au serment. - Si, en revanche, le père avait été tue par un de ses fils, celui-ci devait payer personnellement le «prix de sang augmenté» (diya mugallaza), sans intervention des «contributes solidaires» ${ }^{68}$. Impossible de dire si le fils, Ziyādat Allāh, avait dû payer, après son serment d'innocence, le prix de sang augmenté, mais il a certainement dû participer au dédommagement des mandataires de sang, ses cousins. En revanche, le soupçon de meurtre suffit à déshériter le fils: le fils aîné échappa donc à l'exécution, mais probablement pas à la ruine financière.

Son père, Abū Marwān al-Ṭubnī, la victime dans ce drame familial, était, on l'a dit, un homme riche, malgré les apparences et malgré son comportement d'avare envers sa famille. Après sa mort on trouva que son héritage représentait une grande valeur, enregistrée dans huit cahiers, et qu'il possédait aussi des meubles et des utensiles de quali-

${ }^{67}(\mathrm{IB}(\mathrm{C})), \mathrm{n}^{\circ} 774$.

${ }_{68} \mathrm{Ibn}$ 'Abd al-Barr, $K a \bar{f} f i, 596$. En al-Andalus, le prix de sang augmenté valait 12000 dirham, ibid., 595. 
té, et un grand nombre des vêtements ${ }^{69}$. Pour l'observateur d'aujourd'hui se pose donc la question: qui étaient ses héritiers? ${ }^{70} \mathrm{La}$ loi interdit l'héritage par des personnes qui ont été pardonnées dans le cas de meurtre prémédité. Donc, le fils aîné aurait été déshérité, sa mère-esclave qui n'était pas l'épouse de la victime n'aurait pas hérité légalement, même si elle n'avait pas participé au meurtre. Seuls avraient été héritiers des partages successoraux ( $f a r a ̄ i d)$ dans la famille, de deux neveux et, parenté plus lointaine, les fils de ses oncles paternels, le fils cadet et, peut-être, ses demi-sœurs qui n'étaient pas obligées de prêter le «serment cinquantenaire» ${ }^{71}$.

D'autre part, le motif du meurtre ne fit pas l'objet de la discussion juridique. Les raisons pour lesquelles les femmes avaient tué le maître de maison restent inexpliquées dans le «dossier juridique», c'est à dire dans le récit du juriste Ibn Sahl ${ }^{72}$. On n'a pas non plus établi clairement l'identité des femmes et leur rôle exact dans le drame: actrice, assistante ou victime. Peut-on en déduire que la motivation des acteurs n'influençait pas la discussion juridique occupée uniquement des procédures techniques des preuves, des aveux et des serments? En tout cas, la motivation des acteurs n'était pas considérée comme assez importante pour être transmise à la postérité avec d'autres éléments du cas.

C'est l'historien qui nous fournit avec les détails de la vie familiale d'al-Ṭubnī l'explication de ce drame: le savant n'était pas seulement très avare, il avait délibérément rendu difficile la vie quotidienne de sa famille. Ainsi, il ordonnait que les céréales provenant de ses domaines soient livrés chaque jour en grains; aux femmes de les mouliner de leurs propres mains ${ }^{73}$. Selon une autre source, il ne donnait que du pain sec à sa famille: «s'ils voulaient quelque chose pour accompagner le pain (idām), il devenait furieux» ${ }^{74}$. Après son retour de

${ }^{69}$ (DY) I, 540.

${ }^{70}$ Les sources ne traitent pas cette question.

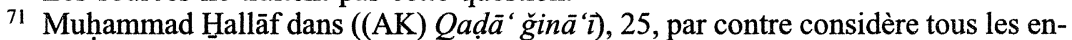
fants comme privés d'héritage, car ils n'étaient pas des wulät al-dam. Pourtant cela ne les exclut pas de l'héritage, sinon les filles n'auraient jamais hérité en cas de mort violente, parce qu'elles étaient privées légalement de l'avantage d'être wali al-dam à l'époque.

72 À l'exception d'une femme qui déclara qu'al-Tubnī «aurait mérité la mort depuis longtemps», citée dans la fatwā d'Ibn 'Attāb ((AK) Qad̄a' ǧinā' $\vec{\imath}), 64$.

73 (DY) I, 537. Cf. aussi Marín, M., Mujeres en al-Andalus (EOBA XI), Madrid, 2000,491 , et son résumé du cas d'al-Tubnī, ibid., 698-700.

${ }^{74}$ Abū Muḥammad al-Hiğāāī, cité dans (IS), I, 93, n. ${ }^{\circ} 26$. 
voyage en Orient, il s'isola de sa famille qu'il avait obligée à résider dans les parties arrières de la maison, plusieurs portes fermées les séparant. Le vieux savant vivait toujours en compagnie de l'adolescent 'Abd al-Raḥmān, dont la maladie étrange a déjà été mentionnée ${ }^{75}$. Sans doute, 'Abd al-Rahmmān a-t-il été reconnu légalement comme le fils d'Abū Marwān, malgré le fait qu'on ignorait l'identité de sa mère et s'il était vraiment le fils biologique d'al-Tubnī. Ces données peuvent expliquer la haine de la famille envers la victime ainsi que le rôle joué par 'Abd al-Raḥmān pendant le procès contre les autres membres de la maison. On a du mal à croire à un meurtre de cupidité. D'autres éléments auraient ajouté à la situation explosive qui aboutit à un meurtre. La mère du fils aîné ainsi que sa femme étaient des esclaves - les sources utilisent les mots umm walad et ğāriya selon le contexte. Donc, elles ne pouvaient pas échapper à leur assujetissement par un divorce. On ignore, pourquoi le jeune couple avec ses enfants n'avait pas quitté la maison paternelle, dans laquelle ils vivaient tranquillement pendant les longues absences du vieux savant en Orient. Ne voulaient-ils pas laisser la mère âgée seule avec ses deux sœurs? Nous avons aussi évoqué la possibilité que la femme de Ziyādat Allāh ait été aussi asservie par le père, ce fait qui aurait déclenché le crime.

Le meurtre n'était donc pas un crime passionnel entraîné par la jalousie envers une autre femme, une esclave rétribuée par exemple: la présence de serviteurs salariés n'est pas mentionnée, mais leur existence dans la maison d'al-Tubnī paraît très improbable étant donné l'avarice de son maître. Y aurait-il eu d'autres membres de la famille vivant hors de la maison du crime? Nos sources ne parlent que des mandataires de sang qui sont toujours des hommes et elles ne mentionnent pas tous les héritiers d'al-Tubnī, femmes et hommes. Il n'est donc pas totalement exclus que le savant ait épousé une femme libre qui vivait hors de la maison du quartier de la Mosquée Amìr, même si cela parait presque impossible étant donné la quantité d'informations dont nous disposons. En tout cas, al-Tubnī n'avait pas d'autres fils que celui mentionné, susceptibles de devenir «mandataires de sang» de leur père. Il est intéressant de noter que l'historien Ibn Hayyān exprime sa compassion pour les victimes du savant poignardé: les fem-

75 Ibn Hayyān dans (DY) I, 537. 
mes auraient tué al-Ṭubnī, poussées au meurtre par son avarice. Il conclut par quelques considérations d'ordre général: "Quelqu'un à qui Dieu donne la richesse ne devrait pas être avare» ou encore: «L'homme est le pasteur responsable de l'entretien de sa famille.» ${ }^{76}$

Pour résumer, nous dirons que c'est la complémentarité des sources qui nous permet d'aller au-delà de l'image officielle du cas judiciaire. Ibn Hayyān, avec l'intérêt qu'il porte aux événements qui se sont déroulés autour des faits juridiques, nous explique comment le premier soupçon du prince est à l'origine de l'enquête policière. De plus, c'est lui, l'historien, qui fournit des détails de nature intime sur la vie familiale dans cette maison fermée, et qui portent atteinte à la réputation du célèbre savant. C'est pourtant la réputation du savant et son rôle dans la transmission du savoir que retient Ibn Pascual/Baškuwāl dans son ouvrage de biographies, le Kitäb al-Sila, tandis qu'il ne fait qu'une brève allusion au meurtre ${ }^{77}$. Le récit d'lbn Hayyän, en revanche, reste incompréhensible car incomplet sans les détails de l'enquête policière et sans les éléments de l'argumentation juridique, telle que les fournit Ibn Sahl. Les deux récits mis ensemble, on comprend mieux le cours des événements et les raisonnements qui mènent le juriste Ibn 'Attāb d'abord, et par la suite le souverain Ibn Ğahwar, à ne pas prendre en compte la peine de hadd et à donner aux inculpés une chance de sortir de prison. Il reste des incohérences dans les données publiées du crime, de petites choses qui nous amènent à penser que l'avarice et les mauvais traitements n'auraient pas été la seule raison de ce meurtre atroce: Quelle haine fallait-il pour donner soixante coups de couteau? Pourquoi la victime ne s'est-elle pas défendue, si ce n'étaient que «ses femmes» l'avaient attaqué? L'une d'elles était-elle si proche de lui physiquement qu'il ne pouvait pas bouger pour se défendre? Et qu'en est-il de ses filles qui auraient participé au crime, mais qui ne doivent pas prêter le serment d'innocence pour se libérer? Et la jeune esclave de la maison, mère des enfants de Ziyädat Allāh, qui selon le fils cadet n'avait pas donné de coups de couteau, pourquoi doit-elle jurer de n'avoir pas participé au meurtre? Il est évident que nos sources nous cachent certaines choses, un élément d'importance peut-étre plus grave encore que l'avarice du père: son

${ }^{76}$ (DY) I, 540.

77 (IB(C)), n. ${ }^{\circ} 772,343-5$; al-Humaydī et Ibn Sa'ĩd ne mentionnent pas ses maitres, cf. $(\mathrm{H}(\mathrm{C}))$, n. $^{\circ} 629,285$, et (IS) I, n. ${ }^{\circ} 26$, p. 92 sq. 
mépris total pour la vie familiale de son fils aîné. Si on considère que le crime avait eu lieu avant ou pendant une soirée amoureuse avec la jeune esclave, les pièces s'ajustent parfaitement. En plus, cela expliquerait d'une façon plus claire pourquoi l'argumentation juridique portait sur le «mort dans le lit conjugal», ainsi en conséquence, pourquoi Ibn 'Attāb n'avait pas accepté le «serment cinquantenaire» des mandataires de sang dans cette affaire, ni par conséquent les indices qui accablaient les inculpés. Le fait que ce soit un crime passionnel aurait ainsi modifié les règles de la doctrine générale.

En conclusion, on peut observer que la violence avec laquelle al-Ṭubnī a été assassiné, n'a pas joué contre les inculpés. L'interrogatoire policier mit en évidence le fait que chacun des inculpés avait participé aux actes de violence à plusieurs niveaux: attendre à la porte de la victime, aider au meurtre et commettre l'acte de tuer. Pourtant, la décision judiciaire ne prit pas compte les différentes formes de violences pour évaluer l'ampleur de la peine: les trois inculpés, y compris le fils aîné qui ne fut pas soupçonné d'avoir levé sa main contre son père, eurent à prêter le même serment afin de sortir de la prison, le degré de violence physique exercé contre la victime ne constituant pas le centre de l'intérêt judiciaire. D'ailleurs, on constate que faits réels et faits juridiques se situent à deux niveaux distincts dans la perception du meurtre. Le serment de «n'avoir pas tué, ni terrassé, ni participé au meurtre» suffit au plan juridique pour que les personnes soupçonnées soient libérées, malgré le fait évident que, dans le cas qui nous intéresse ici, le savant semblait avoir été bel et bien tué par un membre de sa famille. L'obligation de prêter serment constitue de ce fait une forme de violence, non physique, qu'exerce la justice contre les inculpés: ceux-ci, en effet, furent poussés à jurer afin d'éviter de mourir en prison avec le risque évident d'un parjure qui aurait pesé sur leur conscience pendant le reste de leur vie.

\section{ABSTRACT}

La mort violente du savant Abū Marwān al-Ṭubnī en 457/1065 éveilla la curiosité vu les circonstances exceptionnelles de son décès: on l'avait retrouvé dans son lit, percé de plus de soixante coups de poignard, et c'est vers les membres de sa famille, qui vivaient dans diverses parties de la maison, que se portèrent les soupçons. Basé sur un ensemble de sources historiographiques et juridiques, cet article reconstitue les enquêtes menées par les autorités policières 
et les discussions des juristes sur les peines à infliger. Ces données permettent d'analyser les relations au sein d'une famille, relations dont la complexité peuvent expliquer cette violence contre le patriarche.

\section{RESUMEN}

La muerte violenta del sabio Abū Marwān al-Tubnī en 457/1065 despertó una gran curiosidad dadas las circunstancias excepcionales en las que se produjo: al-Ṭubnī fue encontrado muerto en su lecho, atravesado por más de sesenta puñaladas, recayendo sobre los miembros de su propia familia, que vivían en diversas partes de la casa, las principales sospechas. Basado en una serie de fuentes historiográficas y jurídicas, este artículo reconstruye las investigaciones llevadas a cabo por las autoridades policiales así como las discusiones de los juristas sobre los castigos a aplicar. Estos datos permiten analizar las relaciones en el seno de una familia, relaciones cuya complejidad podría explicar la violencia de la que fue objeto el patriarca. 